\title{
Polarization-Sensitive Resonance CSRS of Deoxy- and Oxyhaemoglobin
}

\author{
Artemy Voroshilov, Gerald W. Lucassen, Cees Otto* and Jan Greve \\ Applied Optics Group, Department of Applied Physics, University of Twente, P.O. Box 217, 7500 AE Enschede, The \\ Netherlands
}

\begin{abstract}
Polarization-sensitive coherent Stokes Raman scattering (CSRS) measurements of oxy- and deoxyhaemoglobin in aqueous solutions are reported. The excitation wavelengths used were chosen in the region of the $Q$ absorption bands to achieve twofold electronic resonance. The dispersion profiles of all independent susceptibility $\chi^{(3)}$ components and purely anisotropic and anti-symmetric scattering contributions were resolved within the frequency non-degenerate CSRS scheme. Eight bands of oxyhaemoglobin and five bands of deoxyhaemoglobin were observed in the range $1500-1680 \mathrm{~cm}^{-1}$. Simultaneously fitting sets of polarization spectra provided vibrational parameters (positions, bandwidths, amplitudes, phases and CSRS depolarization ratios) for each compound. Major bands were assigned to the non-totally symmetric $v_{10}, v_{11}$ and $v_{19}$ modes of the porphyrin macrocycle. The phases calculated exhibited a correlation with the symmetry of the vibrations. On the basis of the spectral fits, the three additional peaks arising in the oxyhaemoglobin spectra could be ascribed to the bands of intermediate deoxyhaemoglobin. The occurrence is due to the partial photolysis of oxyhaemoglobin. Vibrational parameters of these bands were found to be essentially similar to the parameters of the bands observed in the spectra of the stable deoxyhaemoglobin. Despite the asymmetric character predicted, the major bands were all contributed to by a considerable isotropic component. $A$ decrease in the depolarization ratio $\rho_{1212}^{R}$ of the anomalously polarized $v_{19}$ mode from 7.7 in oxyhaemoglobin to 4.3 in deoxyhaemoglobin was observed. Such a decrease in anti-symmetric character of the vibration on release of the ligand supports the occurrence of deformation of the haem ring system.
\end{abstract}

\section{INTRODUCTION}

Coherent Raman Spectroscopy (CRS) has proved to be a fruitful ${ }^{1}$ technique for the investigation of vibronic structure and dynamics of biomolecules. Fully exploiting the versatility of frequency non-degenerate CRS may yield information inaccessible with conventional Raman spectroscopy. Specifically, the polarizationsensitive measurements enable one to resolve the dispersion of all susceptibility $\chi^{(3)}$ components $^{2}$ and to detect selectively isotropic, anisotropic and anti-symmetric scattering contributions. The anisotropy of the scattering tensor, which arises due to the enhancement of the non-totally symmetric vibrations, is commonly encountered in applications of resonance Raman spectroscopy. Detailed analysis of the CRS dispersion profiles allows one to derive a complete set of CRS depolarization ratios and, hence, to determine each of the invariants of the Raman polarizability tensor. The latter potential enables one to monitor the vibrational symmetry directly. In addition, the determination of all the CRS depolarization ratios facilitates band assignment, especially in the case of overlapping vibrations. However, in a few reports ${ }^{3-6}$ only, use is made of the possibility of discriminating between $\chi^{(3)}$ components by varying the polarization of incident beams within frequency non-degenerate CRS.

Polarization-sensitive CRS spectroscopy can profitably be applied to studies of biological macromolecules containing chromophoric groups. Particular interest

\footnotetext{
* Author to whom correspondence should be addressed.
}

exists in studies of the nature and function of the haem proteins. These molecules can exhibit a rich resonance enhancement mechanism and a spectral sensitivity to deviations from their idealized $D_{4 h}$ symmetry associated with protein. Numerous spontaneous Raman (SR) studies of haem in a protein matrix ${ }^{7-9}$ particularly haemoglobins, ${ }^{7-17}$ have been conducted under various resonance conditions. With excitation in resonance with the $Q_{00}$ and $Q_{01}$ absorption bands, the haem group displays distinct spectroscopic features such as depolarized and anomalously polarized skeleton vibrations. Thorough analysis has been undertaken to interpret the observed asymmetry of the scattering tensor and the dispersion of the depolarization ratios in terms of haem symmetry distortions. ${ }^{17-19}$ The conformational change $^{20}$ of haem structure on binding and release of ligands has also been monitored. ${ }^{7,10}$ Time-resolved Raman studies of the dynamics of photolysis and allostery in the haem proteins have attracted a great deal of attention. ${ }^{14,15,21}$ Surprisingly, the role of polarizationsensitive CRS in this field is still very limited, ${ }^{22-24}$ probably owing to the absence of haem fluorescence, and therefore the lack of an apparent motivation to use a more complicated technique.

In this paper, the two proximate conformations of a heam in the protein matrix, that is, six- and fivecoordinated haems of human oxyhaemoglobin (oxyHb) and deoxyhaemoglobin (deoxyHb), are examined. Both conformations of the haem are readily classified in the $D_{4 \mathrm{~h}}$ symmetry group. Since their first excited states lie in the visible region, the resonance excitation is easily attainable with tunable dye lasers. Here we report resonance and polarization-sensitive coherent Stokes Raman scattering (CSRS) measurements of non-totally 
symmetric $v_{10}, v_{11}$ and $v_{19}$ haem skeleton modes for both oxy- and deoxyHb molecules excited near the $Q_{00}$ and $Q_{01}$ absorption bands. The spectra of both compounds are analysed and the complete sets of CSRS depolarization ratios are obtained for all vibrations considered. The depolarization ratios reveal the actual symmetry of the scattering tensor, and therefore expose distortions of the haem ring. This paper is intended to show that the application of a coherent technique such as CSRS together with a simultaneous fit of obtained dispersion profiles is an accurate method for characterizing and distinguishing between vibrational bands in the complicated spectra of biological macromolecules.

\section{THEORETICAL CONSIDERATIONS}

In the frequency non-degenerate scheme of the coherent Raman scattering (CRS) the three incident beams having frequencies $\omega_{0}, \omega_{1}$ and $\omega_{2}$, electric field amplitudes $E_{0}, E_{1}$ and $E_{2}$ and linear polarizations $\mathbf{e}_{\alpha}, \mathbf{e}_{\beta}$ and $\mathbf{e}_{\delta}$ respectively, induce the non-linear polarization ${ }^{25,26}$ $\mathbf{P}_{\mu}^{(3)}\left(\omega_{\mathrm{S}}=\omega_{0}+\omega_{1}-\omega_{2}\right)$ at the frequency $\omega_{\mathrm{S}}$. This polarization inspected along $\mathbf{e}_{\mu}$ is related to the components of the third-order susceptibility:

$$
\begin{aligned}
\mathbf{P}_{\mu}^{(3)}\left(\omega_{\mathrm{S}}\right)= & \chi_{\mu \alpha \beta \delta}^{(3)}\left(\omega_{\mathrm{S}} ; \omega_{0}, \omega_{1},-\omega_{2}\right) \\
& \times E_{0}\left(\omega_{0}\right) \mathbf{e}_{\alpha} E_{1}\left(\omega_{1}\right) \mathbf{e}_{\beta} E_{2}{ }^{*}\left(\omega_{2}\right) \mathbf{e}_{\delta}{ }^{*}
\end{aligned}
$$

where the asterisk denotes a photon emission and $\mu, \alpha$, $\beta, \delta=x, y, z$ are the three distinguishable Cartesian coordinates. When analysing the signal field at the frequency $\omega_{\mathrm{S}}=\omega_{0}+\omega_{1}-\omega_{2}$ with a polarizer having a major plane $e_{A}$, the intensity $I_{A}$ detected can be expressed as

$$
I_{\mathbf{A}}\left(\omega_{\mathrm{S}}\right)=\left|\left(\mathbf{e}_{\mathbf{A}}^{*} \cdot \mathbf{P}_{\mu}^{(3)}\left(\omega_{\mathrm{S}}\right)\right)\right|^{2}
$$

If one considers a sample consisting of molecular species, the polarization $\mathbf{P}^{(3)}$ induced consists of the contributions of each group. In the particular case of solvent-solute ensemble the entire polarization consists of the non-vibration resonant polarization of solvent $\mathbf{P}^{(3) N R}$ and the analogous non-vibration resonant (or completely electronic) polarization $\mathbf{P}^{(3) \mathrm{E}}$ and the purely vibration resonant polarization $\mathbf{P}^{(3) \mathbb{R}}$ of the solute. The vibrations of the solvent are not introduced as they are considered weak or distant from the experimental Raman range.

The entire macroscopic molecular susceptibility of the system above can be separated accordingly:

$$
\chi_{\mu \alpha \beta \delta}^{(3)}=\chi_{\mu \alpha \beta \delta}^{(3) \mathrm{NR}}+\chi_{\mu \alpha \beta \delta}^{(3) \mathrm{B}}+\sum_{t} \chi_{\mu \alpha \beta \delta}^{(3) \mathrm{R}}
$$

In contrast to the so-called non-resonant background $\chi_{\mu \alpha \delta \delta}^{(3) \mathrm{NR}}$, which is a real value under the assumption of optical transparency of the solvent, the susceptibility $\chi_{\mu \alpha \beta \delta}^{(3) E}$ may be complex. The phase introduced by $\chi_{\mu \alpha \beta \delta}^{(3) E}$ depends on the proximity of the incident wavenumbers to the electronic resonances of the solute.

The energy diagram depicted in Fig. 1(a) shows the origin of vibration resonant contributions in Eqn (3). The largest resonant terms in the expression for the susceptibility $\chi_{\mu \alpha \beta \delta}^{(3) R}$ can be derived as in Refs 25,27 and 28 . For the particular excitation scheme $|g, 0\rangle \rightarrow \mid k$, $v\rangle \rightarrow|g, t\rangle \rightarrow|j, w\rangle \rightarrow g, 0\rangle$ presented in Fig. 1(a), it

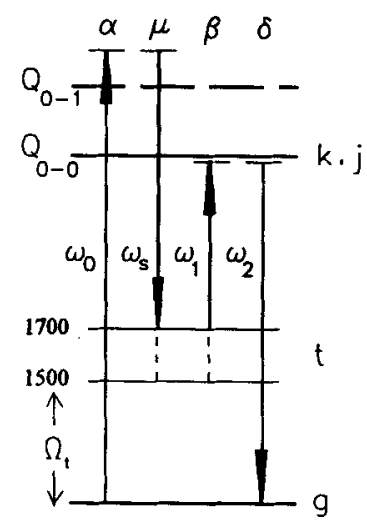

a)

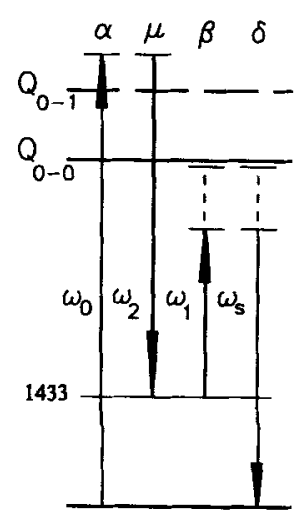

b)
Figure 1. Two major terms contribute to the vibration resonant susceptibility $X_{\mu \alpha \beta \delta}^{(3) \mathrm{R}}$ in the frequency non-degenerate CSRS scheme implemented in the experiments. The electronic ground state is indicated by $g$ and the excited states by $k$ and $\mathbf{j}$. The vibrational states are labelled $t$. Indicies $\alpha, \beta, \delta$ and $\mu$ stand for polarizations of the electric field at wavenumbers $\omega_{0}, \omega_{1}, \omega_{2}$ and $\omega_{s}$, respectively $\left(\omega_{0}>\omega_{2}>\omega_{s}>\omega_{1}\right)$. (a) Resonant (Stokes) contribution $\chi_{\mu \alpha \beta \delta}^{(3)+}\left(\omega_{s} ; \omega_{0}, \omega_{1},-\omega_{2}\right)$ to the non-linear susceptibility; (b) resonant (anti-Stokes) contribution $\chi_{\mu \alpha \beta \delta}^{(3) A}\left(\omega_{5} ; \omega_{1}, \omega_{0},-\omega_{2}\right)$, whose dispersion can be avoided if the difference $\left(\omega_{0}-\omega_{2}\right) / 2 \pi c$ is kept constant at $1433 \mathrm{~cm}^{-1}$.

follows that

$$
\begin{array}{r}
\chi_{\mu \alpha \beta \delta}^{(3) R} \approx \sum_{t} \sum_{k, j} \frac{R_{g k}^{\alpha} R_{k t}^{\mu} R_{i j}^{\beta} R_{j g}^{\delta}}{\left(\Omega_{k}-\omega_{0}-i \Gamma_{k}\right)\left(\Omega_{j}-\omega_{2}+i \Gamma_{j}\right)} \\
\times\left(\Omega_{t}-\left(\omega_{2}-\omega_{1}\right)+i \Gamma_{t}\right)
\end{array}
$$

where indices $\mathbf{k}, \mathbf{j}$ refer to electronic excited states having transition frequencies $\Omega_{k, j}$ and bandwidths $\Gamma_{k, j}$; $\mathrm{g}$ stands for the ground state. The summation runs both over all possible $\mathbf{k}$ and $\mathbf{j}$, as well as all $\mathbf{t}$ vibrations with frequencies $\Omega_{t}$ and bandwidths $\Gamma_{t}$. The matrix element of a transition from the lowest vibrational level 0 in the electronic ground state $|g, 0\rangle$ to the vth vibrational level in the electronic excited state $|k, v\rangle$ is represented by $R_{g k}^{\alpha}=\left\langle k, v\left\|\mu \cdot e_{a}\right\| g, 0\right\rangle$, where $\mu$ is the molecular transition dipole and $\mathbf{e}_{\alpha}$ is the orientation of the electric field. Similar notations for other transitions are used. In deriving Eqn (4), only the ground state is assumed to be populated initially $\left[\rho_{g g}^{(0)}=1\right]$.

The first two denominators in Eqn (4) represent the frequency detunings of applied fields at $\omega_{0}$ (and $\omega_{2}$ ) from the electronic transitions with frequencies $\Omega_{k}$ (and $\Omega_{j}$ ). With $\omega_{0}$ and $\omega_{2}$ both chosen close to the frequency of either of the electronic transitions, the CRS process becomes doubly (electronically) photon resonant. The third (vibrational) denominator contains the resonances of the difference $\left(\omega_{2}-\omega_{1}\right)$ scanned with the $t$ Raman transitions. From Fig. 1(a) and Eqn (4), one can recognize that the CRS process is built up from two Raman pairs, of which the Stokes one acts via the electronic state $\mathbf{k}$ with the product of transition dipole moments $R_{g k}^{\alpha} R_{k t}^{\mu}$ and the anti-Stokes one via electronic state $\mathrm{j}$ with $R_{t j}^{\beta} R_{j g}^{\delta}$. Each pair has its own electronic denominator with resonances at $\boldsymbol{\Omega}_{k}$ and $\boldsymbol{\Omega}_{j}$. Hence, the resonance susceptibility tensor can formally be described as the product of two Raman polarizabilities, each of which contributes with its own enhancement factor. 
When dealing with the non-degenerate third-order process along the scheme $\omega_{\mathrm{s}}=\omega_{0}+\omega_{1}-\omega_{2}$, the susceptibility is contributed to by two principal resonant terms [see Fig. 1(a) and (b)]:

$$
\begin{aligned}
\chi_{\mu \alpha \beta \delta}^{(3) \mathbf{R}}= & \chi_{\mu \alpha \beta \delta}^{(3) \mathbf{R}}\left(\omega_{\mathrm{S}} ; \omega_{0}, \omega_{1},-\omega_{2}\right) \\
& +\chi_{\mu \alpha \beta \delta}^{(3) \mathbf{R}}\left(\omega_{\mathrm{S}} ; \omega_{1}, \omega_{0},-\omega_{2}\right)
\end{aligned}
$$

of which the first involves Raman resonances at the Stokes side and the second at the anti-Stokes side of $\omega_{0}$. If one scans $\omega_{2}$, both terms undergo dispersion and the resulting signal at frequency $\omega_{\mathrm{S}}$ is subject to their interference. In contrast, choosing $\omega_{0}$ and $\omega_{2}$ as fixed and scanning $\omega_{1}$ helps eliminate dispersion of the second term in Eqn (5). Provided that the difference $\left(\omega_{0}\right.$ $-\omega_{2}$ ) coincides with no real vibrations of a sample, the nearly frequency-independent input of the anti-Stokes contribution may be regarded as just an addition to the effective non-resonant background $\chi^{(3) \mathrm{NR}}$. The signal observed is therefore due to Raman resonances at the Stokes side and the entire process may be classified as CSRS. Since $\chi^{(3) R}$ is affected by both electronic resonances of $\omega_{0}$ and $\omega_{2}$ [see Eqn (4)], the influence of electronic denominators in this case is preserved unchanged as the spectrum is scanned. Hence the experimental use of a CSRS scheme instead of CARS one simplifies further spectral analysis.

The susceptibility tensor components are given by an orientational average of the contributions from individual molecules and can be expressed in terms of isotropic $\bar{\alpha}^{2}$, anisotropic $\bar{\gamma}^{2}$ and anti-symmetric $\bar{\gamma}_{\mathrm{A}}^{2}$ invariants of the Raman polarisability tensor. ${ }^{23}$ In isotropic media the $\chi_{\mu \alpha \beta \delta}^{(3)}$ has four distinguishable non-zero components, of which only three are independent and related by

$$
\chi_{1111}^{(3)}=\chi_{1122}^{(3)}+\chi_{1212}^{(3)}+\chi_{1221}^{(3)}
$$

For the CRS depolarization ratios designated as

$$
\begin{array}{ll}
\bar{\rho}=\chi_{1122}^{(3)} / \chi_{1111}^{(3)}, & \bar{\rho}=\chi_{1212}^{(3)} / \chi_{1111}^{(3)}, \\
\rho=\chi_{1221}^{(3)} / \chi_{1111}^{(3)}, & \bar{\rho}+\bar{\rho}+\rho=1
\end{array}
$$

one derives the relationships of CRS depolarization ratios to the Raman invariants above:

$$
\begin{aligned}
& \bar{\rho}^{\mathrm{R}}=\frac{\chi_{1122}^{(3) \mathrm{R}}}{\chi_{1111}^{(3) \mathrm{R}}}=\frac{45 \bar{\alpha}^{2}-2 \bar{\gamma}^{2}}{45 \bar{\alpha}^{2}+4 \bar{\gamma}^{2}} \\
& \bar{\rho}^{\mathrm{R}}=\frac{\chi_{1212}^{(3) \mathrm{R}}}{\chi_{1111}^{(3) \mathrm{R}}}=\frac{5 \bar{\gamma}_{\mathrm{A}}^{2}+3 \bar{\gamma}^{2}}{45 \bar{\alpha}^{2}+4 \bar{\gamma}^{2}} \quad \bar{\rho}^{\mathrm{R}} \geqslant 0 \\
& \rho^{\mathrm{R}}=\frac{\chi_{1221}^{(3) \mathrm{R}}}{\chi_{1111}^{(3) \mathrm{R}}}=\frac{-5 \bar{\gamma}_{\mathrm{A}}^{2}+3 \bar{\gamma}^{2}}{45 \bar{\alpha}^{2}+4 \bar{\gamma}^{2}}
\end{aligned}
$$

The second ratio [Eqn 8(b)] is the CRS analogue of the depolarization ratio $\rho_{\mathrm{SR}}=I_{\perp} / I_{\|}$observable with spontaneous Raman spectroscopy.

As the first non-vibration resonant term in Eqn (3) refers to an optically transparent medium, Kleinman symmetry relationships ${ }^{29}$ hold:

$$
\chi_{1122}^{(3) \mathrm{NR}}=\chi_{1212}^{(3) \mathrm{NR}}=\chi_{1221}^{(3) \mathrm{NR}}=\frac{1}{3} \chi_{1111}^{(3) \mathrm{NR}}
$$

Considering Eqn (6), one derives from Eqn (1)

$$
\begin{array}{r}
\mathbf{e}_{\mathrm{A}}^{*} \mathbf{P}^{(3)} \sim \mathbf{e}_{\mathrm{A}}^{*} \cdot\left[\chi_{1122}^{(3)} \mathbf{e}_{0}\left(\mathbf{e}_{1} \mathbf{e}_{2}^{*}\right)+\chi_{1212}^{(3)} \mathbf{e}_{1}\left(\mathbf{e}_{0} \mathbf{e}_{2}{ }^{*}\right)\right. \\
\left.+\chi_{1221}^{(3)} \mathbf{e}_{2}{ }^{*}\left(\mathbf{e}_{0} \mathbf{e}_{1}\right)\right] E_{0} E_{1} E_{2}{ }^{*}
\end{array}
$$

From Eqn (10), it readily follows that in case of wavenumber degenerate CRS with only two $\left(\omega_{0}=\omega_{1}\right)$ polarized laser beams, the $\chi_{1122}^{(3)}$ and $\chi_{1212}^{(3)}$ are indistinguishable. In contrast, in the case of nondegenerate CRS, employing three separate beams, one is able to discriminate between all the $\chi^{(3)}$ components by simply setting the appropriate polarizations.

Recasting Eqn (4) in a more compressed form gives the expression for the overall susceptibility:

$$
\begin{aligned}
\chi^{(3)}= & p^{\mathrm{NR}} \chi^{(3) \mathrm{NR}}+p^{\mathbf{E}} \chi^{(3) \mathrm{E}} \\
& +\sum_{t} p_{t}^{\mathrm{R}} \frac{A_{t}^{\mathrm{R}} \exp \left(i \Theta_{t}^{\mathrm{R}}\right)}{\left(\mathbf{\Omega}_{t}-\left(\omega_{2}-\omega_{1}\right)+i 2 \Gamma_{t}\right)}
\end{aligned}
$$

with polarization factors defined for non-resonant, electronic and resonant contributions as follows:

$$
\begin{aligned}
p^{\mathrm{NR}, \mathrm{E}, \mathrm{R}}= & \mathbf{e}_{\mathrm{A}} \cdot\left[\bar{\rho}^{\mathrm{NR}, \mathrm{E}, \mathbf{R}} \mathbf{e}_{0}\left(\mathbf{e}_{1} \mathbf{e}_{2}\right)\right. \\
& \left.+\bar{\rho}^{\mathrm{NR}, \mathrm{E}, \mathbf{R}} \mathbf{e}_{1}\left(\mathbf{e}_{0} \mathbf{e}_{2}\right)+\rho^{\mathrm{NR}, \mathrm{E}, \mathbf{R}} \mathbf{e}_{2}\left(\mathbf{e}_{0} \mathbf{e}_{1}\right)\right]
\end{aligned}
$$

and the complexity of electronic susceptibility introduced as $\chi^{(3) E}=a+i b$.

Equations (2), (7) and (9)-(12) form the basis for the computational model used for fitting the spectral data. The numerator in Eqn (11) (usually referred to as $\mathbf{R}+i \mathbf{J}$ in the CARS literature) makes up the total observable vibrational amplitudes $A_{t}^{\mathrm{R}}$ and phases $\Theta_{t}^{\mathrm{R}}$. For each particular vibration these amplitude and phase result from the summation over all $\mathbf{k}$ electronic states involved.

\section{EXPERIMENTAL}

The aqueous solutions of a haemoglobin were prepared from freshly drawn human blood as described in Ref. 22. Erythrocytes were packed by spinning the fresh blood at $2000 \mathrm{~g}$. White blood cells and plasma were then removed. The red cells were washed and lysed against distilled water. Subsequent centrifugation at $25000 \mathrm{~g}$ and the removal of the supernatant provided stable oxyhaemoglobin. The stock oxyHb solution was then dialysed against phosphate buffer ( $\mathrm{pH}$ 7.5). Deoxyhaemoglobin was derived by adding sodium dithionate $\left(\mathrm{Na}_{2} \mathrm{~S}_{2} \mathrm{O}_{4}\right)$ in a slight molar excess to oxyHb solution. Flushing with nitrogen over the solution prevented oxidation of the sample. The stock solutions were monitored spectrophotometrically (Beckman DU8). The concentrations were found to be 3.5 and $3.7 \mathrm{mM}$ for deoxyHb and oxyHb solutions, respectively. ${ }^{12}$ Absorption measurements carried out before, during and after CSRS experiments showed no significant changes.

The CARS/CSRS spectrometer consisted of the two PDL2 dye lasers filled with Rh590 and DCM dyes, which were pumped by the second harmonic of a multimode Nd : YAG laser (Spectra-Physics DCR-2, $10 \mathrm{~Hz}, 8$ ns). Approximately $10 \%$ of the second harmonic was taken as the third beam in the three-colour CSRS experiments. All the beams were led through separate optical channels allowing polarization adjustment of the red $\left(\lambda_{1}=610-650 \mathrm{~nm}\right)$ and yellow $\left(\lambda_{2}=576 \mathrm{~nm}\right)$ beams with double Fresnel rhombs and of the green beam $\left(\lambda_{0}=532 \mathrm{~nm}\right)$ with half-wave retardation plate. Polarizations could be set separately by high-quality Glan- 
Taylor polarizers. The power of each beam (ca. $100 \mu \mathrm{J}$ per pulse) was monitored with the PIN photodiodes for further signal referencing. A lens $(F=250 \mathrm{~mm})$, mounted stress free, focused three beams into the sample to a spot $150-200 \mu \mathrm{m}$ in diameter. The signal beam, analysed with a Glan-Taylor prism, was further dispersed by the double monochromator (modified Jobin Yvon, $200 \mathrm{~mm}$ ) and detected by a photomultiplier tube (Hamamatsu 9973B RCA). Spectra were acquired at room temperature with a spectral resolution $\Delta \omega_{\mathrm{s}} \approx$ $1.2 \mathrm{~cm}^{-1}$ by computer-controlled scans with 50 laser shots per spectral point average. The spectra were corrected for intensity fluctuations and the dye laser efficiency. No data smoothing was applied.

The excitation scheme is displayed in Fig. 1 in the form of an energy diagram and in Fig. 2 by vertical lines. The wavelengths of the second harmonic $\lambda_{0}$ and the yellow dye laser $\lambda_{2}$ were chosen fixed at 532.075 and $576 \mathrm{~nm}$, respectively, so that the wavenumber difference $\left(\omega_{0}-\omega_{2}\right) / 2 \pi c$ equalled $1433 \mathrm{~cm}^{-1}$, which is a Raman region without the strong vibrations of haemoglobin. In this configuration the Raman range $\left(\omega_{1}-\omega_{2}\right) / 2 \pi c$ from -1500 to $-1680 \mathrm{~cm}^{-1}$ could be covered by scanning $\lambda_{1}$ from 630.5 to $638 \mathrm{~nm}$. The signal wavelength varied correspondingly between 578.3 and $584.5 \mathrm{~nm}$.

The sample solution was passed through a flow cuvette (glass wall thickness $2 \times 150 \mu \mathrm{m}$ ) by means of a peristaltic pump. The flow-rate was chosen so as to refresh the volume probed for each laser shot. Multiple interference of the beams on the glass-air boundaries was prevented by employing anti-reflection coated windows. ${ }^{30}$ For the reason of availability, the cuvette length was chosen to be $0.25 \mathrm{~mm}$, being near the optimum value ${ }^{24}$ for the given sample concentrations.

\section{RESULTS AND DISCUSSION}

The absorption spectra of oxyHb and deoxyHb in aqueous solutions in the range $340-645 \mathrm{~nm}$ and the haem chromophore structure are plotted in Fig. 2. The origin of the haem absorption was first described by Gouterman. $^{31}$ The haem electronic structure was further investigated with the help of polarized absorption, $\mathrm{CD}$ and MCD measurements on single crystals of oxyhaemoglobin and deoxylaemoglobin. ${ }^{32-34}$ The bands denoted by Soret (or $B$ ), $Q$ and $N$ all originate from $\pi \rightarrow \pi^{*}$ transitions of the porphyrin macrocycle. Within its ideal $D_{4 h}$ symmetry group the two lowest empty molecular orbitals are degenerate and of $e_{g}\left(\pi^{*}\right)$ symmetry, while the top four filled orbitals occur as nearly degenerate pairs $a_{1 u}(\pi), a_{2 u}(\pi)$ and $a_{2 u}^{\prime}(\pi), b_{2 u}$ $(\pi)$. The transition dipoles of the one-electron promotions $a_{1 u}(\pi) \rightarrow e_{g}\left(\pi^{*}\right)$ and $2_{2 u}(\pi) \rightarrow e_{g}\left(\pi^{*}\right)$ mix to produce the strong $B$ band and the much weaker $Q$ band. The $Q$ band splits into distinct $Q_{00}$ and $Q_{01}$ bands, indicative of the vibronic envelope in the $Q$ state and resulting from the vibronic coupling between $B$ and $Q$ states. $^{8.19}$ Although weak $z$-polarized transitions of metal-porphyrin or metal-metal charge transfer exist, $^{32,33}$ they will not be considered hereafter. As can be seen from Fig. 2, the chosen CSRS excitation allowed us to achieve twofold resonance enhancement owing to the proximity of $\lambda_{2}$ to $Q_{00}$ and of $\lambda_{0}$ to $Q_{01}$ bands.

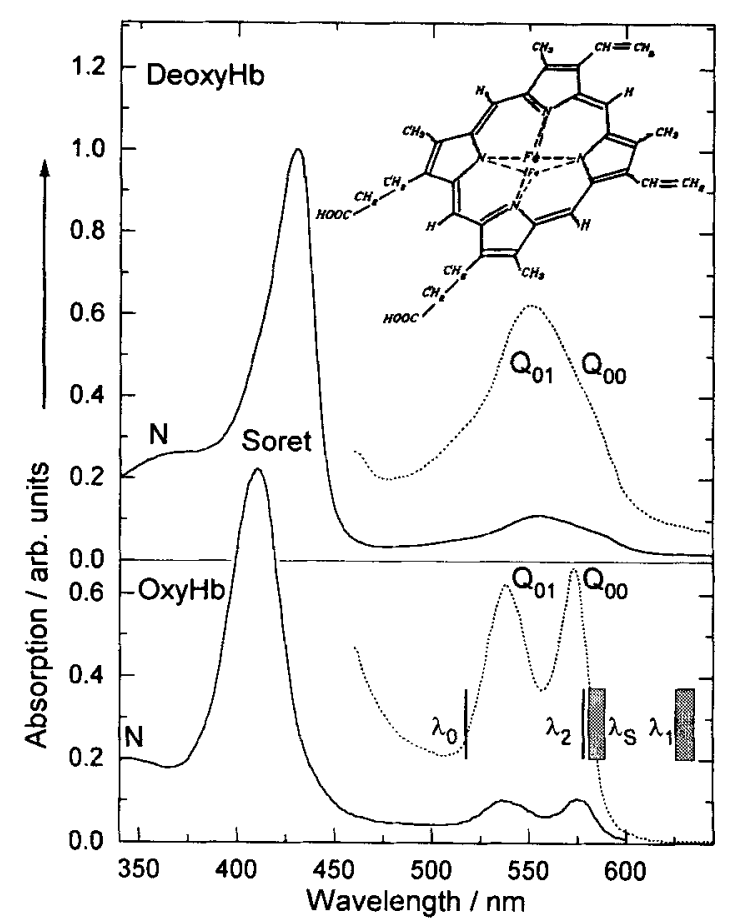

Figure 2. Absorption spectra of aqueous solutions of oxyHb and deoxyHb (pH 7.5). The dotted lines display the scaled absorption near the $Q$ bands. The inset shows the haem structure. The shift of the central iron out of the deoxyHb haem plane, initiated by the release of ligand, is schematically displayed. The wavelength arrangements of the CSRS excitation for probing beams (at $\lambda_{0}$ and $\lambda_{2}$ ) are drawn by vertical lines whereas scanned (at $\lambda_{1}$ and signal (at $\lambda_{s}$ ) beams are indicated by shaded areas.

Figures 3 and 4 show the dispersion profiles of the susceptibility measured (thin lines). For ease of comparison, the corresponding $\chi^{(3)}$ components resolved for oxy- and deoxyHb are plotted next to each other. The columns of plots on the right refer to polarization CSRS measurements on deoxy $\mathrm{Hb}$ and those on the left do so for oxyHb. Figures 3(a)-(c), 3(d)-(f) and $4(a)$ and (d) illustrate the dispersion of $\chi^{(3)}$ components as indicated by the insets. The settings of the polarizers of the incident and signal beams were arranged in accordance with Eqn (10), so as to detect the contribution of each separate $\chi^{(3)}$ term. The spectra given in Fig. 4(b), (e) and (c), (f) demonstrate the possibility of selectively observing anisotropy $\left(\bar{\gamma}^{2} \sim \chi_{1212}^{(3) R}+\chi_{1221}^{(3) R}\right)$ and anti-symmetry $\left(\bar{\gamma}_{\mathrm{A}}^{2} \sim \chi_{1212}^{(3) \mathrm{R}}-\chi_{1221}^{(3) \mathrm{R}}\right)$ of the scattering tensor. The following schemes were applied in each case: $\angle\left(\mathbf{e}_{1}, \mathbf{e}_{0}\right)=$ $45^{\circ}, \mathbf{e}_{1} \perp \mathbf{e}_{2}, \angle\left(\mathbf{e}_{1}, \mathbf{e}_{\mathrm{A}}\right)=45^{\circ}$ and $\angle\left(\mathbf{e}_{1}, \mathbf{e}_{0}\right)=135^{\circ}$, $e_{1} \perp e_{2}, \angle\left(e_{1}, e_{A}\right)=45^{\circ}$. Except for Fig. $4(\mathrm{c})$ and $(f)$, the non-resonant background $\chi^{(3) \mathrm{NR}}$ is present, thus giving rise to complex Raman band shapes via coherent interference. For example, comparing the pair of amplitude spectra in Fig. 3(d) and (f), it is seen that the bands appear as negative instead of positive peaks, showing the opposite signs of the $\chi_{1111}^{(3) R}$ and $\chi_{1122}^{(3) R}$ components with respect to the non-resonant background $\chi^{(3) \mathrm{NR}}$ Similar behaviour can also be seen for the corresponding oxyHb spectra in Fig. 3(a) and (c).

It has been shown for haemoglobins ${ }^{7-12}$ that at the given excitation the contribution of the protein matrix to Raman scattering is negligible and that the bands originating from $C_{\alpha}-C_{m}$ and $C_{\beta}-C_{\beta}$ stretching vibrations of the haem macrocycle dominate the high- 


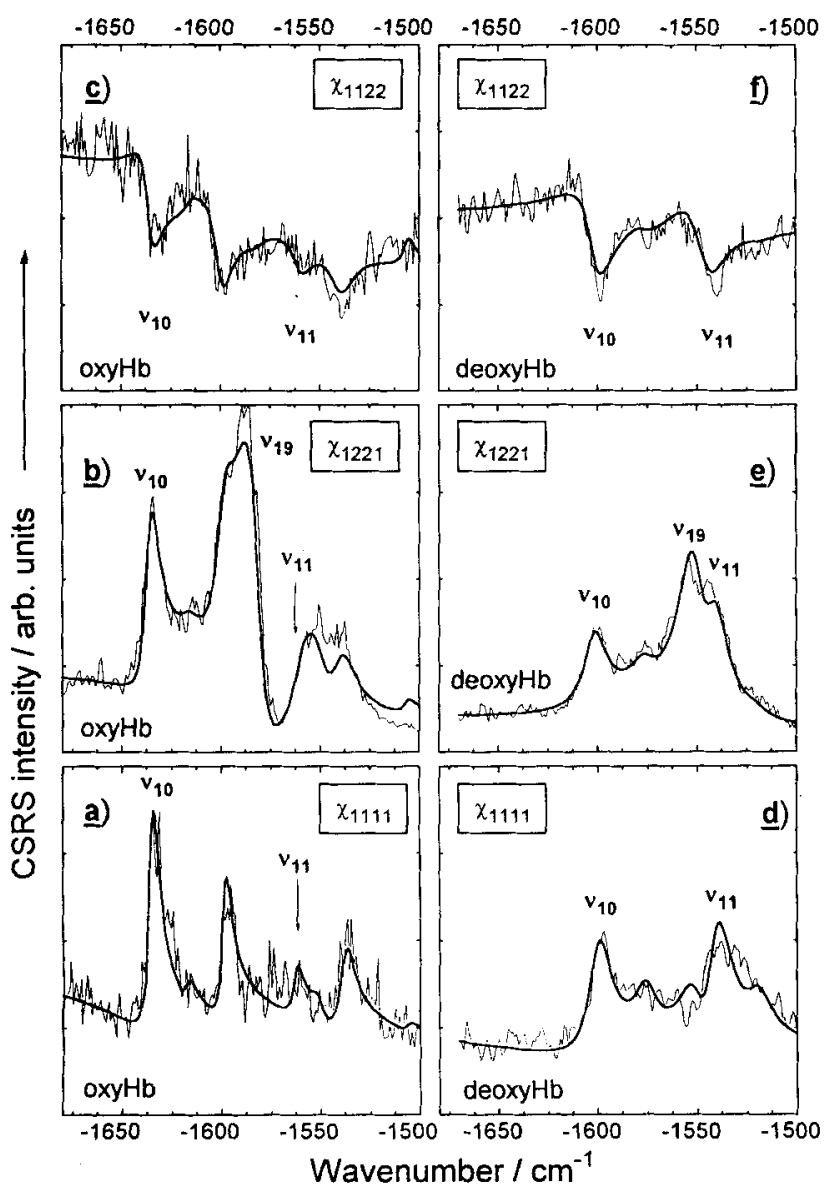

Figure 3. The two sets (a)-(c) and (d)-(e) of polarization CSRS spectra (thin solid lines) of oxyHb and deoxyHb, respectively. The insets indicate which susceptibility component is probed. Thick lines mark the best fits as calculated according to Eqns (11) and (12). In each plot the assignment is given for the most pronounced bands.

frequency Raman spectrum. Presuming that the $D_{4 h}$ representation holds for the symmetry of haem, the skeletal vibrations are classified as $A_{1 g}, B_{1 g}, B_{2 g}$ and $A_{2 g}$ symmetries with Raman depolarization ratios of $1 / 8,3 / 4,3 / 4$ and $\infty$, respectively. Of these modes, the last three are exclusively enhanced if excited near the $Q$ absorption band. Rough classification of the observed band character can be readily undertaken from Figs 3 and 4. For instance, suppression of the non-resonant background in the spectra in Fig. 4(c) and (f) makes it possible to assign easily the anti-symmetrical vibrations. The intense scattering at approximately 1550 and 1580 $\mathrm{cm}^{-1}$ indicates the presence of bands with anomalous polarization, i.e. those of $\boldsymbol{A}_{2 g}$ symmetry. The spectra in Fig. $4(\mathrm{~b})$ and (e) show that the depolarized $B_{1 g}$ bands dominate the region around 1540,1600 and $1635 \mathrm{~cm}^{-1}$. Inspection of Figs 3 and 4 reveals the presence of five Raman bands of deoxyHb and eight of oxyHb observable in the range $1500-1680 \mathrm{~cm}^{-1}$.

With the above considerations, the spectra were fitted according to Eqns (11) and (12), resulting in the best parameter sets listed in Table 1 . The corresponding simulated curves are plotted in Figs 3 and 4 as thick lines. When fitting the spectra we assumed the nonresonant background susceptibility $\chi^{(3) N R}$ to be a real constant. Its depolarization ratios were all taken as $1 / 3$.

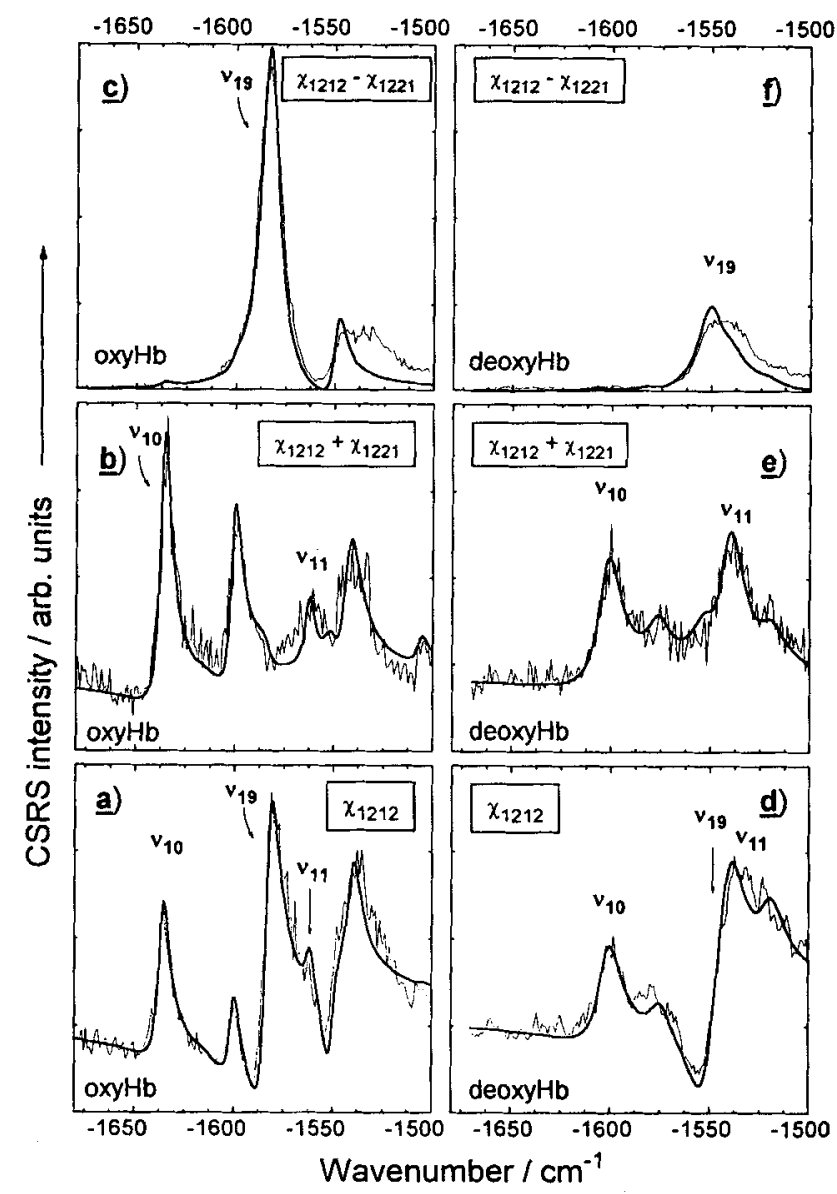

Figure 4. Same as Fig. 3, except for the graphs (b), (e) and (c), $(f)$, where the experimental curves correspond to the anisotropic and the anti-symmetric components of the scattering tensor. Spectra (c) and (f) were obtained with polarization suppression of the non-resonant background (see text).

The analysis of the spectra given in Fig. $4(\mathrm{c})$ and (f) shows a nearly cancelled contribution of the overall background $\chi^{(3) \mathrm{NR}}+\chi^{(3) \mathrm{E}}$ at the edges of the spectra, i.e. the range where there is no input of the vibration resonant $\chi^{(3) R}$. This finding implies that the electronic susceptibility either contributes insignificantly or its depolarization ratios $\bar{\rho}^{\mathrm{E}}, \bar{\rho}^{\mathrm{E}}$ and $\rho^{\mathrm{E}}$ can be taken as equal to $1 / 3$, in which case $\chi^{(3) \mathrm{E}}$ obeys Kleinman symmetry. In such a situation $\chi^{(3) E}$ has no imaginary part and can be incorporated into the real $\chi^{(3) N R}$. A full proof of the possibility of such incorporation can be obtained from a CSRS experiment with polarization suppression of the background with the settings of the polarizers as follows: $\angle\left(e_{1}, e_{0}\right)=120^{\circ}, \angle\left(e_{1}, e_{2}\right)=60^{\circ}$ and $\angle\left(e_{1}, e_{A}\right)=120^{\circ}$. This experiment was conducted for each compound and showed, as in Fig. 4(c) and (f), a negligible background contribution at around 1500 and $1680 \mathrm{~cm}^{-1}$ (results not shown). The CSRS phase are therefore all determined relative to the background which has a zero phase.

It should be emphasized that for each oxy- and deoxyHb, all spectra were simultaneously fitted with the same set of vibrational parameters. Each contribution has its own scaling parameter and linear correction function. The accuracy of fitting is enhanced because more spectra have been measured than the number of 
Table 1. The two groups of vibrational parameters of oxyHb and deoxyHb as obtained from a simultaneous fit on each each of spectra ${ }^{a}$

\begin{tabular}{|c|c|c|c|c|c|c|c|c|c|}
\hline \multirow{2}{*}{$\begin{array}{c}\text { Compound } \\
\text { Oxyhaemoglobin }\end{array}$} & \multicolumn{2}{|c|}{ Assignment $^{3 \mathrm{~B}}$} & \multirow{2}{*}{$\begin{array}{l}\Omega_{d} / \mathrm{cm}^{-1} \\
1618.0\end{array}$} & \multirow{2}{*}{$\begin{array}{c}\Gamma_{/} / \mathrm{cm}^{-1} \\
6.2\end{array}$} & \multirow{2}{*}{$\begin{array}{l}x_{\mathrm{R}}^{(3)} / x_{\mathrm{NR}}^{(3)} \\
0.04\end{array}$} & \multirow{2}{*}{$\begin{array}{r}\Theta_{\gamma^{\circ}} \\
5\end{array}$} & \multirow{2}{*}{$\begin{array}{c}\rho_{1221} \\
0.09\end{array}$} & \multirow{2}{*}{$\begin{array}{l}\rho_{1212} \\
0.14\end{array}$} & \multirow{2}{*}{$\begin{array}{c}\rho_{1122} \\
0.77\end{array}$} \\
\hline & $A_{1 g}$ & $v_{2}, ?$ & & & & & & & \\
\hline & & $v_{3}, ?$ & 1506.0 & 6.5 & 0.04 & 9 & 0.13 & 0.19 & 0.68 \\
\hline & $A_{2 g}$ & $v_{19}$ & 1583.1 & 8.1 & 0.08 & 173 & -6.48 & 7.70 & -0.22 \\
\hline & & $v_{19}^{*} b$ & 1550.7 & 7.8 & 0.07 & 127 & -2.68 & 3.97 & -0.29 \\
\hline & $B_{1 g}$ & $v_{10}$ & 1635.9 & 7.1 & 0.45 & 47 & 0.58 & 0.72 & -0.30 \\
\hline & & $v_{10}^{*} b$ & 1600.5 & 7.3 & 0.33 & 35 & 0.52 & 0.68 & -0.20 \\
\hline & & $v_{11}$ & 1562.0 & 6.0 & 0.14 & 54 & 0.57 & 0.62 & -0.19 \\
\hline & & $v_{11}^{*}{ }^{b}$ & 1540.0 & 8.0 & 0.23 & 48 & 0.65 & 0.73 & -0.38 \\
\hline Deoxyhaemoglobin & $A_{1 g}$ & $v_{2}, ?$ & 1575.9 & 7.0 & 0.06 & 55 & 0.53 & 0.55 & -0.08 \\
\hline & & $v_{3}, ?$ & 1518.7 & 7.3 & 0.13 & 95 & 0.54 & 0.55 & -0.09 \\
\hline & $A_{2 g}$ & $v_{19}$ & 1550.2 & 8.0 & 0.05 & 124 & -3.04 & 4.28 & -0.24 \\
\hline & $B_{1 g}$ & $v_{10}$ & 1601.8 & 7.0 & 0.17 & 43 & 0.59 & 0.70 & -0.29 \\
\hline & & $v_{11}$ & 1542.2 & 7.8 & 0.18 & 47 & 0.59 & 0.82 & -0.41 \\
\hline
\end{tabular}

a Fitting parameters include Raman line positions, bandwidths, amplitudes (normalized to the one of a background), phases and CSRS depolarization ratios.

${ }^{b}$ Asterisks indicate the modes attributed to deoxyHb which is formed due to the partial photolysis of oxyHb. The modes $v_{19}^{*}, v_{10}^{*}$ and $v_{11}^{*}$ correspond to the $v_{19}, v_{10}$ and $v_{11}$ modes, respectively, of stable deoxyHb listed in the bottom part (see text).

independent susceptibilities. Considering the uncertainties in obtaining parameters from the curve-fitting procedure, the inaccuracy of the depolarization ratios is estimated as $c a .5 \%$.

Considering the analogy of depolarization ratios $\bar{\rho}^{\mathbf{R}}=\chi_{1212}^{(3) \mathrm{R}} / \chi_{1111}^{(3) \mathrm{R}}$ to $\mathrm{SR}$ ratios $\rho_{\mathrm{SR}}=I_{\perp} / I_{\|}$, the major bands, i.e. those at 1583,1636 and $1562 \mathrm{~cm}^{-1}$ of oxyHb and 1550,1602 and $1542 \mathrm{~cm}^{-1}$ of deoxyHb, can be firmly assigned to the $v_{19}, v_{10}$ and $v_{11}$ haem skeleton modes, respectively. ${ }^{35}$ Although weaker deoxyHb bands at 1519 and $1576 \mathrm{~cm}^{-1}$ (and those of oxyHb at 1506 and $1618 \mathrm{~cm}^{-1}$ ) were incorporated into the fit, their assignment to polarized $v_{3}$ and $v_{2}$ modes, respectively, remains ambiguous.

A comparison of the spectra in Figs 3 and 4 shows a correspondence of the bands of oxy- and deoxyhaemoglobin at 1551,1600 and $1540 \mathrm{~cm}^{-1}$. The results of the fits for both molecules reveal that not only positions of the oxyHb modes denoted $v_{19}^{*}, v_{10}^{*}$ and $v_{11}^{*}$ (see Table 1) but also their vibrational phases and depolarization ratios accord well with those of the deoxyHb modes $v_{19}, v_{10}$ and $v_{11}$. It is therefore concluded that anomalously polarized and two depolarized bands found for oxyHb at 1551,1600 and $1540 \mathrm{~cm}^{-1}$ should be assigned to the deoxyHb modes $v_{19}, v_{10}$ and $v_{11}$, respectively.

The dynamics of the photolysis and the further relaxation of the protein surrounding the haem cavity contain a number of stages distinguished on the femtosecond to picosecond time scale. ${ }^{14}$ On photolysis, the oxyhaem releases a ligand and undergoes a transition to the iron-shifted (or domed) deoxyhaem configuration. This occurs on a subpicosecond scale ${ }^{21}$ and is accompanied by the immediate $(0.35 \mathrm{ps})$ development of deoxyhaem absorption spectrum. ${ }^{15}$ Therefore, from the outset is was clear that the CRS measurments with nanosecond time resolution would only reveal the mixture of the longest lived species. Specifically with the incident laser beam energies applied, the molecules of the oxyHb molecuies are photolysed, ${ }^{22}$ resulting in the formation of the species of deoxyhaem. The experimental data and the theoretical modelling of the spectra show nicely the contribution of more than one molecular species to the CSRS spectra. It should be emphasized that more important for this study is the conclusion that the full characterization of each vibrational band can be reliably obtained by the procedure described above, which is a unique feature of a coherent technique with respect to spontaneous Raman spectroscopy.

Direct comparison of the CSRS and SR depolarization ratios is complicated as the former are affected by multiple electronic resonances with the wavenumbers of incident beams. However, a calculated depolarization ratio $\bar{\rho}^{\mathrm{R}}$ of 7.7 for the $v_{19}$ mode of oxyHb is consistent with that of 10 reported for resonance Raman spectra excited at the centre of the $Q_{00}$ band. $^{8}$ On the other hand, the ratio $\bar{\rho}^{\mathrm{R}}$ obtained deviates from its expected value of $\infty$ under ideal $D_{4 h}$ symmetry, indicating [Eqn (8)] a considerable isotropy $\bar{\alpha}^{2}$ of the scattering tensor involved. This result agrees well with a proposed model ${ }^{17}$ that considers symmetry classified normal quasi-static distortions of the haem ring. Such perturbations introduce new components into the Raman tensor by reducing the haem symmetry. The partially isotropic character of the $v_{19}$ haem mode is further supported by the value of $\bar{\rho}^{R}=\chi_{1122}^{(3) R} / \chi_{111}^{(3) R}=$ -0.22 for this band. A value $\bar{\rho}^{R}=-0.5$ would be expected in the absence of a symmetric contribution to the scattering. In lowering the oxyhaem symmetry, which occurs when the bound oxygen is released, the anomalously polarized $v_{19}$ mode also gains some anisotropy, which is manifested by its ratio $\bar{\rho}^{R}$ decreasing even further down to 4.28 . The increase in anisotropy can be seen in Fig. $4(\mathrm{e})$ and suggests an admixture of asymmetric (or $B_{1 g} / B_{2 g}$ ) scattering contributions due to an analogous haem distortion.

A comparison of spectra representing the dispersion of $\chi_{1111}^{(3) R}$ components [Fig. 3(a) and (d)] and the anisotropy $\bar{\gamma}^{2}$ [Fig. $4(\mathrm{~b})$ and (e)] in the first place reveals a similar pattern. This is evidence for the prevalence of the anisotropic component in the scattering. However, on closer examination, for most of the pronounced bands (i.e. $v_{19}, v_{10}$ and $v_{11}$ ) the values of $\bar{\rho}^{\mathrm{R}}$ range from -0.2 to -0.4 instead of -0.5 , thus indicating the exis- 
tence of an isotropic contribution. In addition, the contrast between the ratios $\rho_{1221}^{R}$ and $\bar{\rho}_{1212}^{R}$ of, for instance, the depolarized $v_{10}$ modes is seen, which indicates the presence of a small anti-symmetric contribution. These ratios should be identical in the absence of an antisymmetric (or $A_{2 g}$ ) scattering component. The comparison of the depolarization ratios obtained for the $v_{11}$ modes also reveals their partially anti-symmetric character. On going from oxy- to deoxyhaem, the difference between $\rho_{1221}^{\mathrm{R}}$ and $\bar{\rho}_{1212}^{\mathrm{R}}$ ratios of the $v_{11}$ mode even rises from 0.05 to 0.23 , evidently exhibiting a growth of the anti-symmetric component, associated with the increasing distortion of the haem ring.

To the authors' knowledge, the vibrational phases are seldom accounted for in the CRS literature, even though Eqn (4) postulates that phases contain information on the mechanisms of resonance enhancement and vibronic coupling. Well away from electronic resonances the influence of the summation over the different electronic states $\mathbf{k}$ and $\mathbf{j}$ can be regarded as resulting from a single distant electronic state and the vibrational phases are all close to zero because of real electronic denominators in Eqn (4). In contrast, the vibrational phases rise as the wavelength of excitation approaches electronic transition. When enhancement of $\chi^{(3) R}$ is produced due to the (pre-)resonance of the single exciting wavenumber with a single electronic transition, which has a fixed transition dipole moment, the vibrational phases follow the phase introduced by the only (electron-)resonant denominator. At this point the vibrational depolarization ratios are generally expected $^{36}$ to be around $1 / 3$ as there should be no antisymmetry of scattering tensor involved. In the case that both $\omega_{0}$ and $\omega_{2}$ are (pre-)resonant to the single electronic transition, the vibrational phases are contributed to by the two unequal complex denominators ${ }^{27}$ in Eqn (4). Such excitation, however, does not influence the magnitudes of depolarization ratios. The situation becomes complicated, though, if the CRS excitation occurs near the manifold of absorption bands that have different orientations of transition dipole moments. Vibrations may then be differently coupled to electronic transitions $\mathbf{k}$ and $\mathbf{j}$. The varying coupling strengths included into products $R_{g k}^{\alpha} R_{k t}^{\mu}$ and $R_{i j}^{\beta} R_{j g}^{\delta}$ in Eqn (4) therefore determine the specific weights with which a particular electronic state affects the phase of a vibration. In such a case the phase may vary from one vibration to another. Further, each vibration may couple excited electronic states [vibronic coupling, omitted in Eqn (4)]. The unequal coupling also causes a deviation of the depolarization ratios from $1 / 3$. Anomalously polarized bands obtain their intensity, at least partially, from an anti-symmetric scattering tensor, which in turn arises due to vibronic coupling. Hence the $\chi^{(3) R}$ incorporates several electronic enhancement factors which can be different for the modes with differing symmetries.

It can be seen from Table 1 that anomalously polarized $v_{19}$ modes have clearly distinct phases $\Theta_{t}>120^{\circ}$, whereas the phases of all the depolarized $B_{1 g}\left(v_{11}\right.$ and $v_{10}$ ) bands tend to vary between $35^{\circ}$ and $55^{\circ}$. Such phase correlations conform with the hypothesis of the symmetry-dependent coupling of the vibronic transitions to the electronic transitions. At this point it seems difficult to distinguish between the mechanisms of vibrational phase variations mentioned above or, better, to decompose the total vibrational phases and amplitudes into electronic contributions. A detailed analysis of the phase correlations may prove useful for the interpretation of the non-linear CRS spectra. Such an analysis requires a detailed knowledge of the molecular electronic structure.

\section{CONCLUSIONS}

We have reported the first application of polarizationsensitive non-degenerate CSRS to porphyrins in a protein matrix. Excitation near the $Q$ absorption bands resulted in resonant enhancement of the non-totally symmetric $v_{10}, v_{11}$ and $v_{19}$ modes of both oxy- and deoxyhaemoglobin. A fitting program was developed that allowed the simultaneous fit of each set of polarization spectra. Consistent sets of band parameters (including positions, bandwidths, amplitudes, phases and depolarization ratios) were derived for both oxyand deoxyHb. The capability of CRS technique to distinguish between the vibrational contributions of various species to the scattering has been shown on the example of the decomposition and assignment of the bands of partially photolysed oxyHb. The bands of photoproduct were found to correspond identically with the bands of stable deoxyHb. The analysis of calculated depolarization ratios was carried out in terms of Raman tensor invariants that indicated asymmetric distortions: of the haem ring. The deviations from $D_{4 h}$ symmetry: were found to increase little on going from oxy- to deoxyhaem. The remarkable correlation of the vibrational coherent phase and symmetry of vibration has. been detected.

\section{Acknowledgement}

The authors are indebted to Y. Kraan for preparation of the samples.

\section{REFERENCES}

1. G. L. Eesley, Coherent Raman Spectroscopy. Pergamon Press, New York (1981); W. M. Tolles and A. B. Harvey, in Chemical Applications of Nonlinear Raman Spectroscopy, edited by A. B. Hanvey. p. 1. Academic Press, New York (1981); S. A. J. Druet and J.-P. E. Taran, Prog. Quantum Electron. 7, 1(1981).

2. S. A. Akhmanov, A. F. Bunkin, S. G. Ivanov and N. I. Koroteev, Sov. Phys. JETP 47, 667 (1978); B. Dick, Chem. Phys. 113,131 (1987).

3. P. A. Apanasevich, V. V. Kvach, V. P. Kozich and V. A. Orlovich, J. Appl. Spectrosc. 38, 699 (1983).
4. B. N. Toleutaev, PhD Thesis, Moscow State University (1989).

5. W. Li, H.-G. Purucker and A. Laubereau, Opt. Commun. 94, 300 (1992)

6. H.-G. Purucker, V. Tunkin and A. Laubereau, J. Raman Spectrosc. 24, 453 (1993).

7. T. G. Spiro and T. C. Strekas, J. Am. Chem. Soc. 96, 338 (1974).

8. R. Schweitzer-Stenner and W. Dreybrodt, J. Raman Spectrosc. 16, 111 (1985) 
9. S. Choi, J. J. Lee, Y. H. Wei and T. G. Spiro, J. Am. Chem. Soc. 105, 3692 (1983).

10. T. G. Spiro, Isr. J. Chem. 21, 81 (1981).

11. R. Schwezer-Stenner, W. Dreybrodt, A. Mayr and S. el Naggar, J. Raman Spectrosc. 13, 139 (1982).

12. E. Antonini and M. Brunori, Haemoglobin and Myoglobin in Their Reactions with Ligands. North-Holland, Amsterdam (1971)

13. R. Schwezer-Stenner, D. Wedekind and W. Dreybrodt, Biophys. J. 49, 1077 (1986).

14. L. Genberg, L. Richard, G. McLendon and R. J. D. Miller, Science 251, 1051 (1991).

15. T. G. Spiro, G. Smulevich and C. Su, Biochemistry, 29, 4497 (1990).

16. M. C. Hsu and R. W. Woody, J. Am. Chem. Soc. 93, 3615 (1971).

17. R. Schwezer-Stenner, U. Dannemann and W. Dreybrodt, Biochemistry 31, 694 (1992).

18. M. Z. Zgierski and M. Pawlowski, Chem. Phys. 65, 335 (1982).

19. R. Schweitzer-Stenner, U. Bobinger and W. Dreybrodt, J. Raman Spectrosc. 22, 65 (1991).

20. M. F. Perutz, Annu. Rov. Phys. Chem. 48, 327 (1979).

21. S. Franzen, B. Bohn, C. Poyart and J.-L. Martin, in Pro ceedings of the VI Conference on Time Resolved Vibrational Spectroscopy, edited by A. Lau, F. Siebert and W. Werncke, p. 199. Springer, Berlin (1993).
22. R. F. Dallinger, J. R. Nestor and T. G. Spiro, J. Am. Chem. Soc. 100, 6251 (1978).

23. R. Igarashi, Y. Adachi and S. Maeda, J. Chem. Phys. 72, 4308 (1980).

24. T. A. H. N. Scholten, PhD Thesis, University of Twente, Enschede (1989).

25. Y. Prior, IEEE J. Quantum Electron. 20,37 (1984).

26. H. Lotem, R. T. Lynch and N. Bloembergen, Phys. Rev. A 14, 1748 (1976)

27. S. A. J. Druet, A. A. Attal, T. K. Gustafson and J. P. Taran, Phys. Rev. A 18, 1529 (1978).

28. S. Maeda, T. Kamisuki, H. Kataoka and Y, Adachi, Appl. Spectrosc. Rev. 21, 211 (1985)

29. D. A. Kleinman, Phys. Rev. 126, 1977 (1962).

30. T. A. H. M. Scholten, G. W. Lucassen, F. F. M. de Mul and J. Greve, Appl. Opt. 27, 3225 (1988).

31. M. Gouterman, J. Chem. Phys. 30. 1139 (1959).

32. M. W. Makinen and W. A. Eaton, Anal. N. Y. Acad. Sci. 206 210 (1973).

33. A. K. Churg and M. W. Makinen, J. Chem. Phys. 68, 1913 (1978).

34. W. A. Eaton, L. K. Hanson, P. J. Stephens, J. C. Sutherland and J. B. R. Dunn, J. Am. Chem. Soc. 100, 4991 (1978).

35. M. Abe, T. Kitagava and Y. Kyogoku, J. Chem. Phys. 69, 4526 (1978).

36. J. Behringer, in Raman Spectroscopy, edited by H. A. Szymanski. p. 205. Plenum Press, New York (1967) 